\title{
GLR-Entropy Model for ECG Arrhythmia Detection
}

\author{
M. Ali Majidi ${ }^{1}$ and H. SadoghiYazdi ${ }^{1,2 *}$ \\ ${ }^{1}$ Department of Computer Engineering, Ferdowsi University of Mashhad, \\ Mashhad, Iran \\ ${ }^{2}$ Center of Excellence on Soft Computing and Intelligent Information Processing, \\ Ferdowsi University of Mashhad, Iran \\ ali.majidianvari@stu.um.ac.ir,h-sadoghi@um.ac.ir
}

\begin{abstract}
In this paper a novel unsupervised classification method for electrocardiogram (ECG) signal classification is presented. The proposed approach classifies the input signal into normal and abnormal heartbeat patterns with a relatively high accuracy. After extracting features from the time-voltage waves in ECG signals, we utilize a computationally fast algorithm based on log likelihood strategy for change detection on selected features. We then combine the outputs based on their validation coefficient. The Algorithm could differentiate between the normal and unknown heart features. Experimental results show the accuracy of the proposed approach in terms of reliability and performance.
\end{abstract}

Keywords: Electrocardiogram (ECG), Generalized Likelihood Ratio (GLR), change detection, Entropy

\section{Introduction}

The electrocardiogram (ECG) is a low cost, and effective test for arrhythmia analysis and has become the standard diagnostic tool. An important step toward identifying an arrhythmia is the classification of heartbeats. The classification of an electrocardiogram (ECG) into different disease categories is a complex pattern recognition task. Classification of heartbeats can be very time-consuming and hence any automated processing of the ECG that assists this process would be of assistance and is the focus of this study.

Conventionally, a typical heart beat is identified from the ECG and the component waves of the QRS, T and possibly $\mathrm{P}$ waves are characterized using measurements such as magnitude, duration and area. Datasets that are used for heart diseases involve different features. Some of them are based on laboratory experiments, while others include clinical symptoms. However, one of the most popular and useful databases is the MIT-BIH.

Automated classification of heartbeats has been previously reported by other researchers. Several methods have been proposed for the classification of ECG signal. Classifiers methods employed include linear discriminants [1], back propagation neural networks [2,3], self-organizing maps with learning vector quantization [4], and selforganizing networks [5].

In [5] a method is described for clustering ECG heartbeats from a recording into 25 clusters and determined that on average $98.5 \%$ of the heartbeats in any one cluster were

${ }^{*}$ H. SadoghiYazdi is the corresponding author (E-mail: h-sadoghi@um.ac.ir) 
from the same heartbeat class. This translates to a classification performance of $98.5 \%$ if an expert can correctly identify the dominant beat of a cluster. In [6] a SVM based method for PVC arrhythmia detection shown that has a better efficient rather than ANFIS. In [7] a new approach based PSO-SVM has been proposed for feature selection and classification of cardiac arrhythmias. In [8], a neuro-fuzzy approach for the ECGbased classification of heart rhythms is described. Here, the QRS complex signal is characterized by Hermite polynomials, whose coefficients feed the neuro-fuzzy classifier. Detection of arrhythmia by means of Independent Component Analysis (ICA) and wavelet transform to extract important features is proposed in [9]. Finally, in [10], the authors present an approach for classifying beats of a large dataset by training a neural network classifier using wavelet and timing features. The authors found that the fourth scale of a dyadic wavelet transform with a quadratic spline wavelet together with the pre/post RR-interval ratio is very effective in distinguishing normal and PVC from other beats. The paper is structured as follows. In Section 2 preliminaries is explained. This section covers an overview of Generalized Likelihood Ratio (GLR) method and Shannon Entropy. The structure of the proposed change detector is discussed in details in Section 3. The effectiveness of the proposed approach is illustrated by experimental results in Section 4. Finally, Section 5 presents the concluding remarks and future work.

\section{Preliminaries}

\subsection{Generalized Likelihood Ratio (GLR) Change Detector}

GLR algorithm [11] is based on a concept that is very important in mathematical statistics, namely the logarithm of the likelihood ratio, defined by:

$$
\mathrm{s}(\mathrm{y})=\ln \frac{\mathrm{p}_{\theta_{1}}(\mathrm{y})}{\mathrm{p}_{\theta_{0}}(\mathrm{y})}
$$

And referred to as the log-likelihood ratio.

The key statistical property of this ratio is as follows: Let $E_{\theta_{0}}$ and $E_{\theta_{1}}$ denote the expectations of the random variables under the two distributions $p_{\theta_{0}}$ and $p_{\theta_{1}}$, respectively. Then,

$$
\mathrm{E}_{\theta_{0}}<0 \text { and } \mathrm{E}_{\theta_{1}}>0
$$

In other words, a change in the parameter $\theta$ is reflected as a change in the sign of the mean value of the log-likelihood ratio. This property can be viewed as a kind of detectability of the change.

Log-likelihood ratio for the observations from time $\mathrm{j}$ up to time $\mathrm{k}$ is:

$$
S_{j}^{k}=\sum_{i=j}^{k} s_{i}=\sum_{i=j}^{k} \ln \frac{p_{\theta_{1}}\left(y_{i}\right)}{p_{\theta_{0}}\left(y_{i}\right)}
$$

In the present case $\theta_{1}$ is unknown; therefore, this ratio is a function of two unknown independent parameters: the change time and the value of the parameter after change. The standard statistical approach is to use the maximum likelihood estimates of these two parameters, and thus the double maximization:

$$
\mathrm{g}_{\mathrm{k}}=\max _{1 \leq \mathrm{j} \leq \mathrm{k}} \ln \widehat{\Lambda}_{\mathrm{j}}^{\mathrm{k}}=\max _{1 \leq \mathrm{j} \leq \mathrm{k}} \sup _{\theta_{1}} \mathrm{~S}_{\mathrm{j}}^{\mathrm{k}}\left(\theta_{1}\right)
$$

Now let us discuss further the issue of level of available a priori information about the parameter after change. In many applications, it is possible to know a minimum 
magnitude $\mathrm{v}_{\mathrm{m}}$ of the changes of interest in the parameter $\theta$. In this case, the second maximization in the GLR algorithm can be achieved using this minimum magnitude of change as follows:

$$
\mathrm{g}_{\mathrm{k}}=\max _{1 \leq \mathrm{j} \leq \mathrm{k}} \sup _{\theta_{1}:\left|\theta_{1}-\theta_{0}\right| \geq \mathrm{v}_{\mathrm{m}}>0} S_{\mathrm{j}}^{\mathrm{k}}\left(\theta_{1}\right)
$$

Let us return consider of change in the mean of an independent Gaussian sequence. In this case, the mean $\mu_{0}$ before change is known, and the mean $\mu_{1}$ after change is unknown. The constant variance $\sigma^{2}$ is also known. The corresponding cumulative sum can be rewritten as

$$
S_{j}^{k}=\frac{\mu_{1}-\mu_{0}}{\sigma^{2}} \sum_{i=j}^{k}\left(y_{i}-\frac{\mu_{1}+\mu_{0}}{2}\right)
$$

Let us introduce $\mathrm{v}=\mu_{1}-\mu_{0}$ then:

$$
\mathrm{g}_{\mathrm{k}}=\max _{1 \leq \mathrm{j} \leq \mathrm{k}} \sup _{\mathrm{v}:|\mathrm{v}| \geq \mathrm{v}_{\mathrm{m}}>0} \sum_{\mathrm{i}=\mathrm{j}}^{\mathrm{k}}\left(\frac{\mathrm{v}\left(\mathrm{y}_{\mathrm{i}}-\mu_{0}\right)}{\sigma^{2}}-\frac{\mathrm{v}^{2}}{2 \sigma^{2}}\right)
$$

In the present independent Gaussian case, the constrained maximization over $\mathbf{v}$ is explicit:

$$
\mathrm{g}_{\mathrm{k}}=\max _{1 \leq \mathrm{j} \leq \mathrm{k}} \sum_{\mathrm{i}=\mathrm{j}}^{\mathrm{k}}\left(\frac{\widehat{v}\left(\mathrm{y}_{\mathrm{i}}-\mu_{0}\right)}{\sigma^{2}}-\frac{\mathrm{v}^{2}}{2 \sigma^{2}}\right)
$$

Where the absolute value of the constrained change magnitude estimate is:

$$
\left|\widehat{v}_{j}\right|=\left(\frac{1}{k-j+1} \sum_{i=j}^{k}\left|y_{i}-\mu_{0}\right|-v_{m}\right)^{+}+v_{m}
$$

And its sign is the same as the sign of the mean value $\frac{1}{\mathrm{k}-\mathrm{j}+1} \sum_{\mathrm{i}=\mathrm{j}}^{\mathrm{k}}\left(\mathrm{y}_{\mathrm{i}}-\mu_{0}\right)$ of the last centered observations or "innovations."

Note also that, when $\mathrm{v}_{\mathrm{m}}=0$ the decision function is:

$$
\mathrm{g}_{\mathrm{k}}=\frac{1}{2 \sigma^{2}} \max _{1 \leq \mathrm{j} \leq \mathrm{k}} \frac{1}{\mathrm{k}-\mathrm{j}+1}\left[\sum_{\mathrm{i}=\mathrm{j}}^{\mathrm{k}}\left(\mathrm{y}_{\mathrm{i}}-\mu_{0}\right)\right]^{2}
$$

By applying an appropriate threshold on $g_{k}$, we can detect the changes (If it exists).

\subsection{Shannon Entropy}

Suppose $\mathrm{X}$ is random variable which takes on a finite set of values according to a probability distribution $\mathrm{p}(\mathrm{X})$. Based on [12] the entropy of this probability distribution is defined to be the quantity:

$$
H(X)=\sum_{i=1}^{n} p\left(x_{i}\right) \log _{b} \frac{1}{p\left(x_{i}\right)}=-\sum_{i=1}^{n} p\left(x_{i}\right) \log _{b} p\left(x_{i}\right)
$$

Where $\mathrm{X}$ takes the value $x_{i}, 1 \leq i \leq n$.

\section{The Proposed Method}

Main motivation in this paper is ECG arrhythmias detection with used GLR algorithm that its reliability increases by Shannon entropy. Our method inputs are signals that each of them belong to a feature of heart beats. Each of these signals has two parts: The first part is made of a feature that extracted of a healthy heart beats. We called this part as normal samples. The second part includes corresponding feature with first part that extracted of an unknown heart beats (i.e., healthy or sick heart). We called this part as abnormal samples. We will used first and second parts of each input signal 
as training data and test data respectively. The aim is detection arrhythmia if second part belong to a sick heart beats. All of features, extracted by a feature extraction method that in this paper we used a method that proposed on [13]. In Figure 1 shows an input signal that belong to PQ feature.

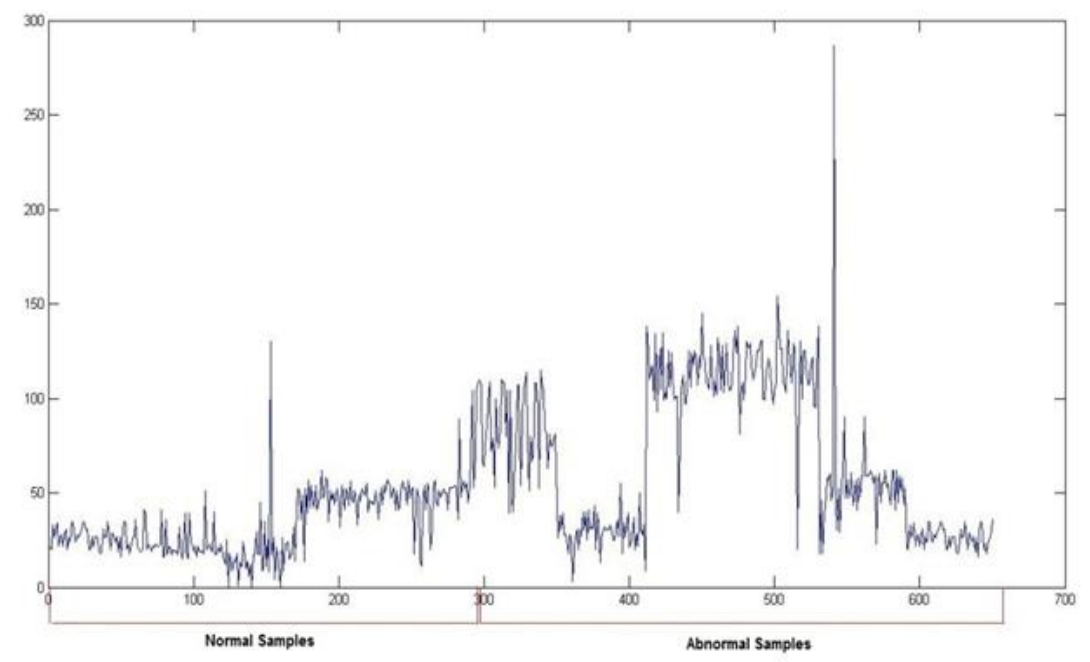

Figure 1. PQ feature for 290 healthy beats and 360 unhealthy beats

Now we explain our method: in train stage, for each input signal, run GLR algorithm on normal samples. Then Shannon entropy of GLR algorithm output is calculated and used as reliability to output's GLR algorithm. We named it as confidence coefficient of each feature. In test stage first run GLR algorithm on abnormal samples. Then used confidence coefficient to combining results of GLR algorithms. At the end, used an appropriate threshold on main output (combined output) for arrhythmia detection if it exist. In fact our method combine variant feature that extracted of ECG signal based on confidence coefficient to heart arrhythmias detection. Also we can used this method to change point detection of heart activity. In the following, the stages and pseudo code of our method is shown.

\section{Train stage:}

- Feature extraction of a healthy heart ECG.

- Run GLR algorithm on normal samples.

- Compute confidence coefficients.

\section{Test stage:}

- Feature extraction of unknown heart ECG.

- Run GLR algorithm on abnormal samples.

- Combining GLR algorithm output based on their confidence coefficients.

- Threshold on main output. 


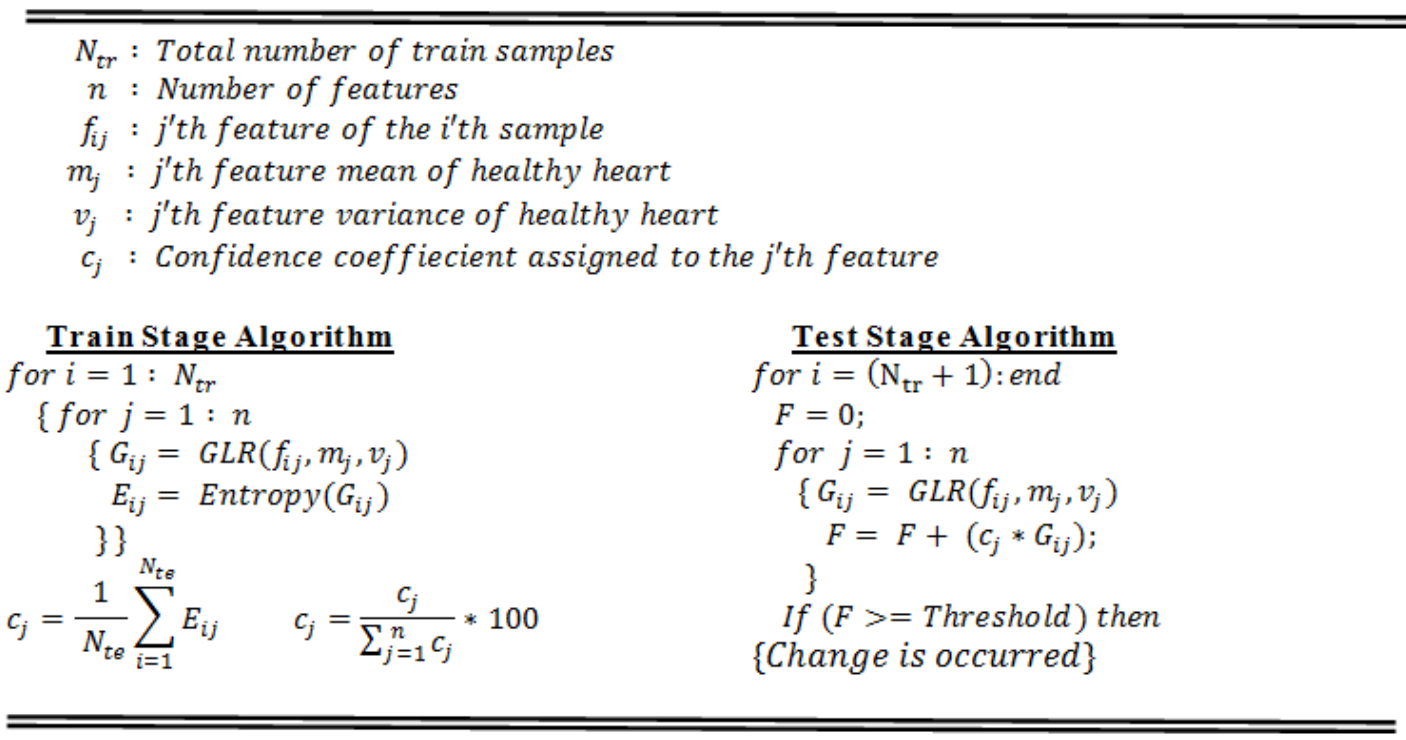

Where $\operatorname{GLR}($, , ) and Entropy () are functions that calculated the outputs of GLR algorithm and Shannon Entropy that explained in Preliminaries section respectively. The value of $m_{j}$ and $v_{j}$ can be calculated by averaging in a dataset of healthy heart beats.

\section{Discussion}

Sequence beats of healthy heart usually have fixed mean and variance for features such as PQ interval, ST interval, $S$ voltage, $T$ voltage, $Q$ voltage, etc. that could be calculated based on expert notation or feature extraction algorithms. When an arrhythmia occur, mean or variance of these features will be changed which can be detected by a constant threshold. But when some features are not extracted correctly, accuracy of this method reduced significantly. For this reason we used of GLR algorithm. When features are extracted correctly, GLR output is constant so its entropy is low and reliability is high but if features are extracted with many errors, its entropy is high and reliability is low. We used this property for compute confidence coefficients. Note that confidence coefficients compute from features that extracted of healthy heart beats.

\section{Experimental Result}

In this paper, the ECG records, available at the MIT-BIH arrhythmia database are used. The database has 48 records with each record being an ECG signal for the duration of 30 minutes. Each data was recorded in two channels, modified limb lead II and modified lead VI. In particular, the considered beat types refer to following classes: normal sinus rhythm $(\mathrm{N})$, right bundle branch block (RB), left bundle branch block (LB), and paced beat $(\mathrm{P})$. The beats were selected from the recording of following patients, 100, 106, 107, 109, 118, 202, 209,212, 214, 215 and 217.

We run proposed method on 290 normal and 360 abnormal samples.

The results are shown in the Figure 2. As shown in this figures, feature 'ST' has lower entropy than feature 'PT' in normal samples, so feature 'ST' has greater confidence coefficient. 


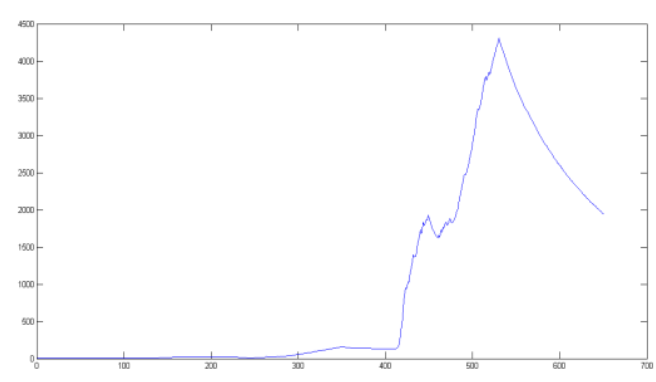

(a)

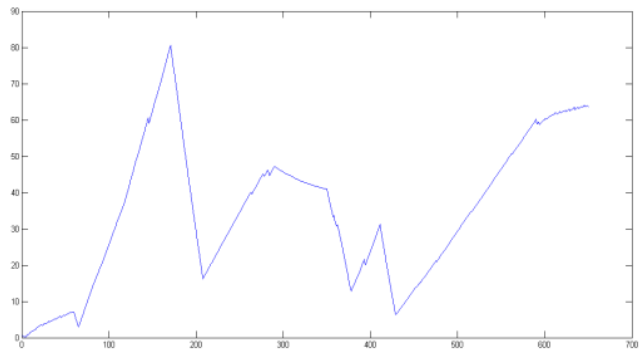

(c)

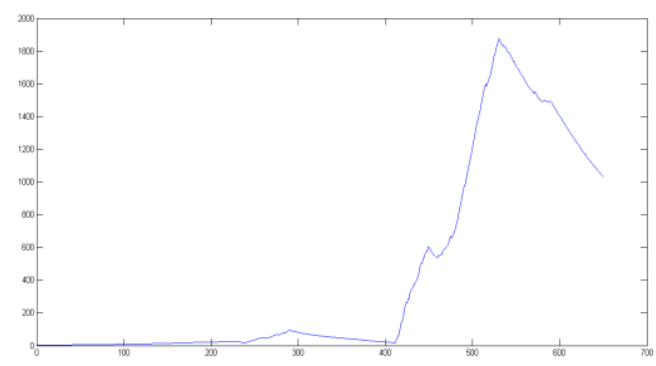

(e)

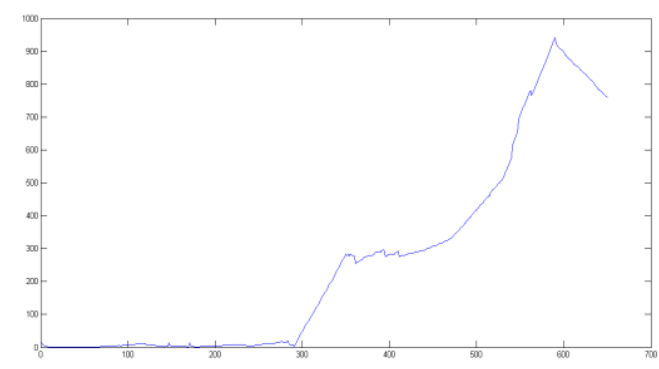

(g)

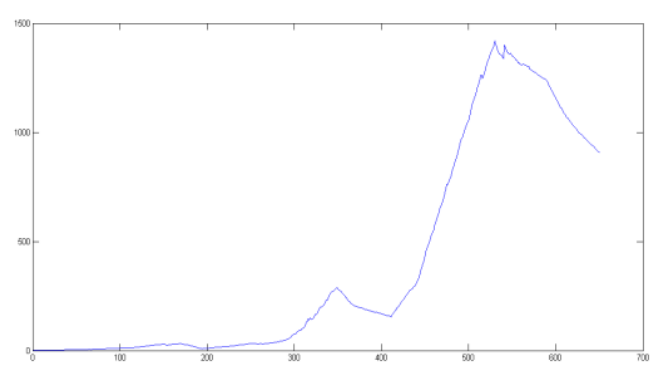

(b)

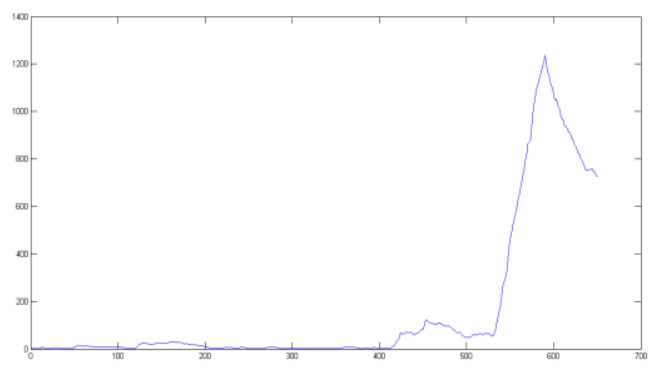

(d)

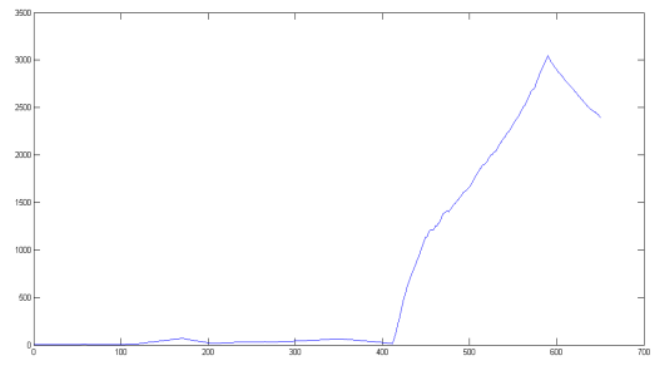

(f)

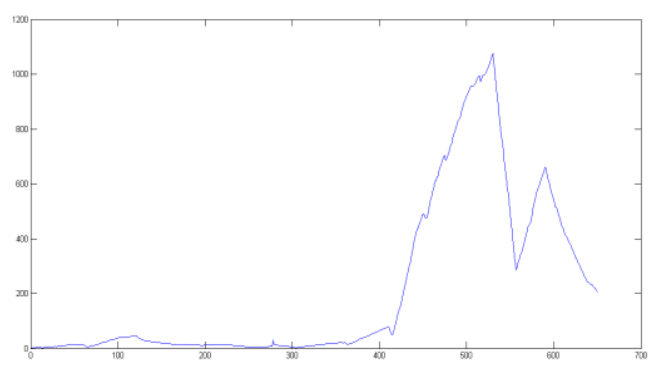

(h)

Figure 2. Run GLR Algorithm on 8 feature: (a) 'P voltage', (b) 'PQ interval', (c) 'PT interval', (d) 'Q voltage', (e) 'RP interval', (f) 'S voltage', (g) 'ST interval' and (h) 'T voltage'

Final output is showed in Figure 3 that combine above features based on their coefficient.

In final stage we should select a conveniently threshold. In this case if it is equal 25 then change point select currently on time 290 . 


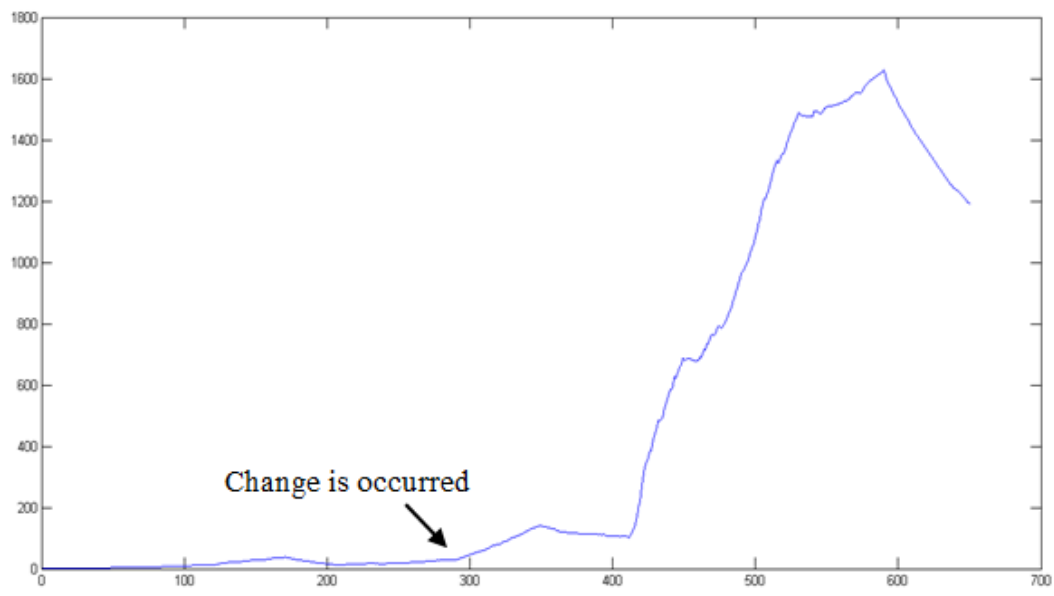

\section{Figure 3. Output of GLR-Entropy algorithm for 290 normal sample and 360 abnormal}

\section{Conclusion}

In this paper, a new method is proposed based on GLR change detection algorithm, entropy and Multi Features Data Fusion (MFDF) strategy for electrocardiogram (ECG) signal classification. Experimental results show good classification on normal and abnormal samples.

\section{References}

[1] L. Senhadji, G. Carrault, J. J. Bellanger and G. Passariello, "Comparing wavelet transforms for recognizing cardiac patterns", Engineering in Medicine and Biology Magazine, IEEE, vol. 14, (1995), pp. 167-173.

[2] T. Yeap, F. Johnson and M. Rachniowski, "ECG beat classification by a neural network", in Engineering in Medicine and Biology Society, 1990., Proceedings of the Twelfth Annual International Conference of the IEEE, (1990), pp. 1457-1458.

[3] S. Osowski and T. H. Linh, "ECG beat recognition using fuzzy hybrid neural network", Biomedical Engineering, IEEE Transactions on, vol. 48, (2001), pp. 1265-1271.

[4] Y. H. Hu, S. Palreddy and W. J. Tompkins, "A patient-adaptable ECG beat classifier using a mixture of experts approach", Biomedical Engineering, IEEE Transactions on, vol. 44, (1997), pp. 891-900.

[5] M. Lagerholm, C. Peterson, G. Braccini, L. Edenbrandt and L. Sornmo, "Clustering ECG complexes using Hermite functions and self-organizing maps", Biomedical Engineering, IEEE Transactions on, vol. 47, (2000), pp. 838-848.

[6] A. Gharaviri, F. Dehghan, M. Teshnelab and H. Abrishami Moghaddam, "Comparison of neural network, ANFIS, and SVM classifiers for PVC arrhythmia detection", in Machine Learning and Cybernetics, 2008 International Conference on, (2008), pp. 750-755.

[7] F. Melgani and Y. Bazi, "Classification of electrocardiogram signals with support vector machines and particle swarm optimization", Information Technology in Biomedicine, IEEE Transactions on, vol. 12, (2008), pp. 667-677.

[8] S. O. A. M. L. S. T. H. Linh, "On-line heart beat recognition using Hermite polynomials and neuro-fuzzy network " IEEE TRANS INSTRUM MEAS, vol. 52, (2003), pp. 1224-1231.

[9] A. Azemi, V. R. Sabzevari, M. Khademi, H. Gholizade, A. Kiani and Z. S. Dastgheib, "Intelligent arrhythmia detection and classification using ICA", in Engineering in Medicine and Biology Society, 2006. EMBS'06. 28th Annual International Conference of the IEEE, (2006), pp. 2163-2166.

[10] O. T. Inan, L. Giovangrandi and G. T. Kovacs, "Robust neural-network-based classification of premature ventricular contractions using wavelet transform and timing interval features", Biomedical Engineering, IEEE Transactions on, vol. 53, (2006), pp. 2507-2515.

[11] M. E. Basseville and I. V. Nikiforov, "Detection of abrupt changes: theory and application," (1993).

[12] R. M. Gray, Entropy and information theory: Springer, (2011). 
[13] H. S. Yazdi, "JDL Fusion Model for ECG Arrhythmia Detection," International Journal of Signal Processing, Image Processing and Pattern Recognition, vol. 5, (2012).

\section{Authors}

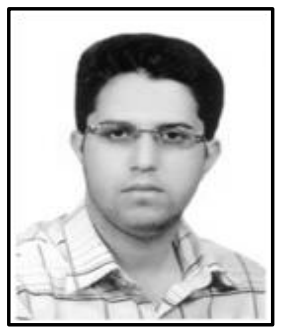

\section{Ali Majidi}

He received his B.Sc. degree in Sadjad Institute of Higher Education of Mashhad, Iran in 2010. He is currently a M.Sc. student at the Computer Science and Engineering Department at Ferdowsi University of Mashhad (FUM). He is studying Signal Processing and Pattern Recognition under the supervision of H. Sadoghi. His research interests include pattern recognition, machine learning, unknown input filtering and change detection.

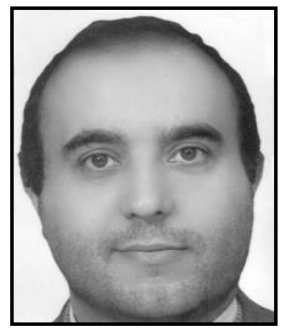

\section{Hadi Sadoghi Yazdi}

$\mathrm{He}$ is currently an Associate Professor of Computer Science and Engineering at Ferdowsi University of Mashhad (FUM). He received his B. S. degree in Electrical Engineering from FUM in 1994, and received his M.S. and Ph.D. degrees in Electrical Engineering from Tarbiat Modares University in 1996 and 2005, respectively. Dr. SadoghiYazdi has received several awards including Outstanding Faculty Award and Best System Design Award in 2007. His research interests are in the areas of Pattern Recognition, Machine Learning, Machine Vision, Signal Processing, Data Mining and Optimization. 